\title{
The effect of toe marker placement error on joint kinematics and muscle forces using OpenSim gait simulation
}

\author{
Hang $\mathrm{Xu}^{\mathrm{a}, \mathrm{b}, *}$, Andrew Merryweather ${ }^{\mathrm{b}}$, Donald Bloswick ${ }^{\mathrm{b}}$, Qi Mao ${ }^{\mathrm{a}}$ and Tong Wang ${ }^{\mathrm{a}}$ \\ ${ }^{a}$ Department of Medical Imaging, Xuzhou Medical College, 209 Tongshan Road, Xuzhou, P.R. China \\ ${ }^{b}$ Department of Mechanical Engineering, University of Utah, 50 S Central Campus Dr, Salt Lake City, \\ $U T, U S A$
}

\begin{abstract}
Marker placement can be a significant source of error in biomechanical studies of human movement. The toe marker placement error is amplified by footwear since the toe marker placement on the shoe only relies on an approximation of underlying anatomical landmarks. Three total knee replacement subjects were recruited and three self-speed gait trials per subject were collected. The height variation between toe and heel markers of four types of footwear was evaluated from the results of joint kinematics and muscle forces using OpenSim. The reference condition was considered as the same vertical height of toe and heel markers. The results showed that the residual variances for joint kinematics had an approximately linear relationship with toe marker placement error for lower limb joints. Ankle dorsiflexion/plantarflexion is most sensitive to toe marker placement error. The influence of toe marker placement error is generally larger for hip flexion/extension and rotation than hip abduction/adduction and knee flexion/extension. The muscle forces responded to the residual variance of joint kinematics to various degrees based on the muscle function for specific joint kinematics. This study demonstrates the importance of evaluating marker error for joint kinematics and muscle forces when explaining relative clinical gait analysis and treatment intervention.
\end{abstract}

Keywords: Toe marker placement, gait simulation, joint kinematics, muscle force

\section{Introduction}

The fundamental role of human movement analysis is to better understand the physiopathology of musculoskeletal system. Stereophotogrammetry is usually recruited to undertake this task of collecting raw data for this analysis. However, some errors exist when using stereophotogrammetry, at least in three aspects: (1) the instrumental errors associated with stereophotogrammetric system; (2) marker movement caused by the skin deformation and displacement; and (3) the misplacement of anatomical landmark [1-3]. Evaluation and estimation of these errors are crucial for assessing the precision and reliability of human movement analysis and prevent to misexplain the relative results, such as joint kinetics and kinematics.

\footnotetext{
*Address for correspondence: Hang Xu, Department of Medical Imaging, Xuzhou Medical College, 209 Tongshan Road, Xuzhou, P.R. China. Tel.: +86 5168326 2243; Fax: +86 5168326 2162; E-mail: h_xu@xzmc.edu.cn.
}

0959-2989/15/\$35.00 @ 2015 - IOS Press and the authors. 
Gait analysis is widely used to investigate normal and pathological gait to describe how human walks, and has significant clinical value to define treatment programs for abnormal gait [4]. The most commonly applied method of gait analysis is performed by tracking clusters of reflective markers placed on the skin to identify various anatomical landmarks. These markers are used to reconstruct body segments and to define orientations of segments in space and time. However, marker placement error exists and causes up to about $75 \%$ failures of kinematic parameters during the period of marker capture [5]. Previous research focused on marker placement error for different cases. Szczerbik and Kalinowska found that lower limb joint kinematics were significantly altered when knee marker position was changed in a systematic way [6]. O'Connor et al. indicated that marker placement had a significant effect on measuring the range of motion of spinal flexion/extension and lateral sidebending [7]. France and Nester suggested that the quadriceps angle was sensitive to the error of identifying anatomical landmarks and the center of the patella [8].

In practice, it is very common that foot markers are placed on footwear instead of underlying anatomical landmarks during gait. The marker placement error is amplified in this situation because the toe marker position only relies on the best approximation as shown in Figure 1. Since joint kinematics were effected by marker placement and play a great role in the prediction of muscle forces using marker-driven musculoskeletal models [9]. It is important to know how much marker placement error affects estimation of joint kinematics and predicted muscle forces when evaluating individual muscle functionality and treatment intervention. Therefore, the purpose of this study was to evaluate the effect of toe marker placement in the vertical direction on lower limb joint kinematics and muscle forces during gait.

\section{Methods}

\subsection{Experiment data}

Three total knee replacement males (age $81 \pm 2$ years, height $173 \pm 6 \mathrm{~cm}$, and mass $70 \pm 4 \mathrm{~kg}$ ) were the subjects for this study. The subjects were asked to walk at a self-speed pace and were give a pair of flat bottom sneaker. For each subject, three normal gait trials were collected, which included markerbased video motion and ground reaction data [10]. The marker trajectory followed a modified Cleveland clinic marker set. The toe and heel markers were put at the same height on the sneaker, like the standard barefoot gait marker set requested and considered as the reference condition in this study. Marker motion was measured using a 10-camera motion capture system (Motion Analysis Corp., Santa Rosa, CA) and Ground reaction forces was measured using three force plates (AMTI Corp., Watertown, MA).

In order to evaluate practical toe marker placement error in the vertical direction, four types of footwear, including work shoe, sport shoe, leather shoe and walking shoe, were chosen to determine a common range of height difference between heel and toe markers. The heel and toe markers were carefully put on the best approximation of the posterior calcaneus and center of the foot between the 2nd and 3rd metatarsals on the surface of each shoe. The height difference (h) between the heel and toe markers in the sagittal plane was measured using a 3D motion analysis system (NaturalPoint Inc., Corvalis, OR) calibrated with sub-millimeter accuracy $(<0.5 \mathrm{~mm}$ residual error). The different $\mathrm{h}$ values were $5.5 \mathrm{~cm}$ for work shoe, $3.0 \mathrm{~cm}$ for sport shoe, $1.5 \mathrm{~cm}$ for leather shoe and $1.0 \mathrm{~cm}$ for walking shoe, respectively. 


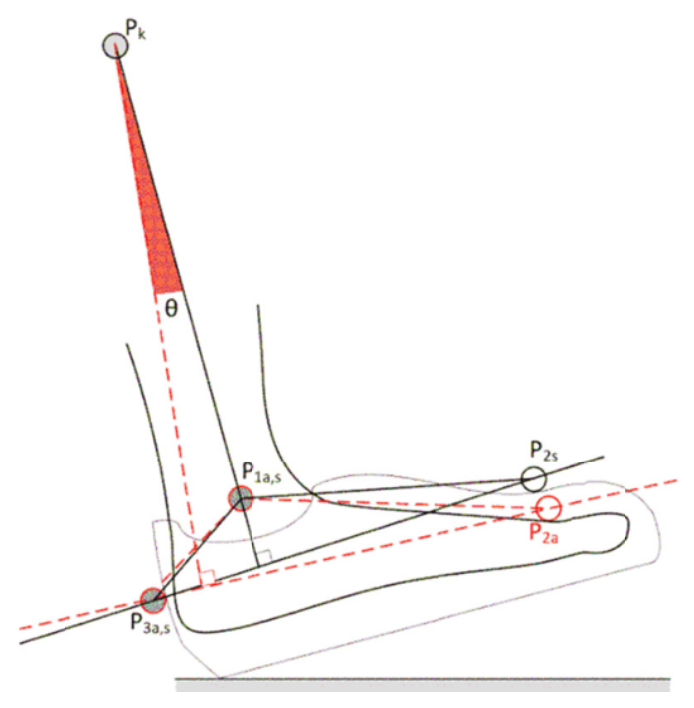

Fig. 1. Toe marker placement error. The toe markers $\mathrm{P}_{2 \mathrm{a}}$ and $\mathrm{P}_{2 \mathrm{~s}}$ represented anatomical position and the approximate positions on a shoe respectively. $\theta$ represented the ankle angle error in the sagittal plane caused by the error in the placement of these markers.

\subsection{Gait simulation}

An OpenSim model was recruited in this study, which consisted of 12 body segments, 23 degrees of freedom (DOF) and 54 muscles [11]. The hip was represented as a three DOF ball-and-socket joint, the knee was represented as a single DOF hinge joint in the sagittal plane with proximodistal and anteroposterior translations occurring as a function of knee flexion. The ankle joint was represented as a single DOF universal joint. The model was firstly scaled to match the subject's height and weight. Then segment lengths were obtained from the marker positions acquired during the static trial, and segment masses were scaled based on anthropometric data and subject's body weight.

Major gait events (heel strike and toe off) were defined via force plate activation with a $20 \mathrm{~N}$ threshold to identify the gait cycle. Three self-speed gait trials for each subject were firstly used to simulate the reference situation $(\mathrm{h}=0 \mathrm{~cm})$ in one gait cycle. Then, the toe marker on the model was adjusted in the vertical direction to match the various height difference (h) identified by marker placements on the footwear, and the three trials for each subject were resimulated to assess the effect of toe marker position error. During OpenSim gait simulation, the joint kinematics was determined with a weighted least squares solution and muscle forces were reported based on the computed muscle control algorithm [12].

\subsection{Data process}

The results of lower limb joint kinematics (hip, knee and ankle) and muscle forces (16 muscles) in knee placement limb were firstly normalized by 101 data point, then averaged among trials and subjects compared between four height differences and $\mathrm{h}=0$. Normalized root mean square error (NRMSE) was used to describe the residual variance. The formulas for NRMSE were shown in Eqs. (1) and (2). Results were considered significant difference when NRMSE above 10\% [13]. 

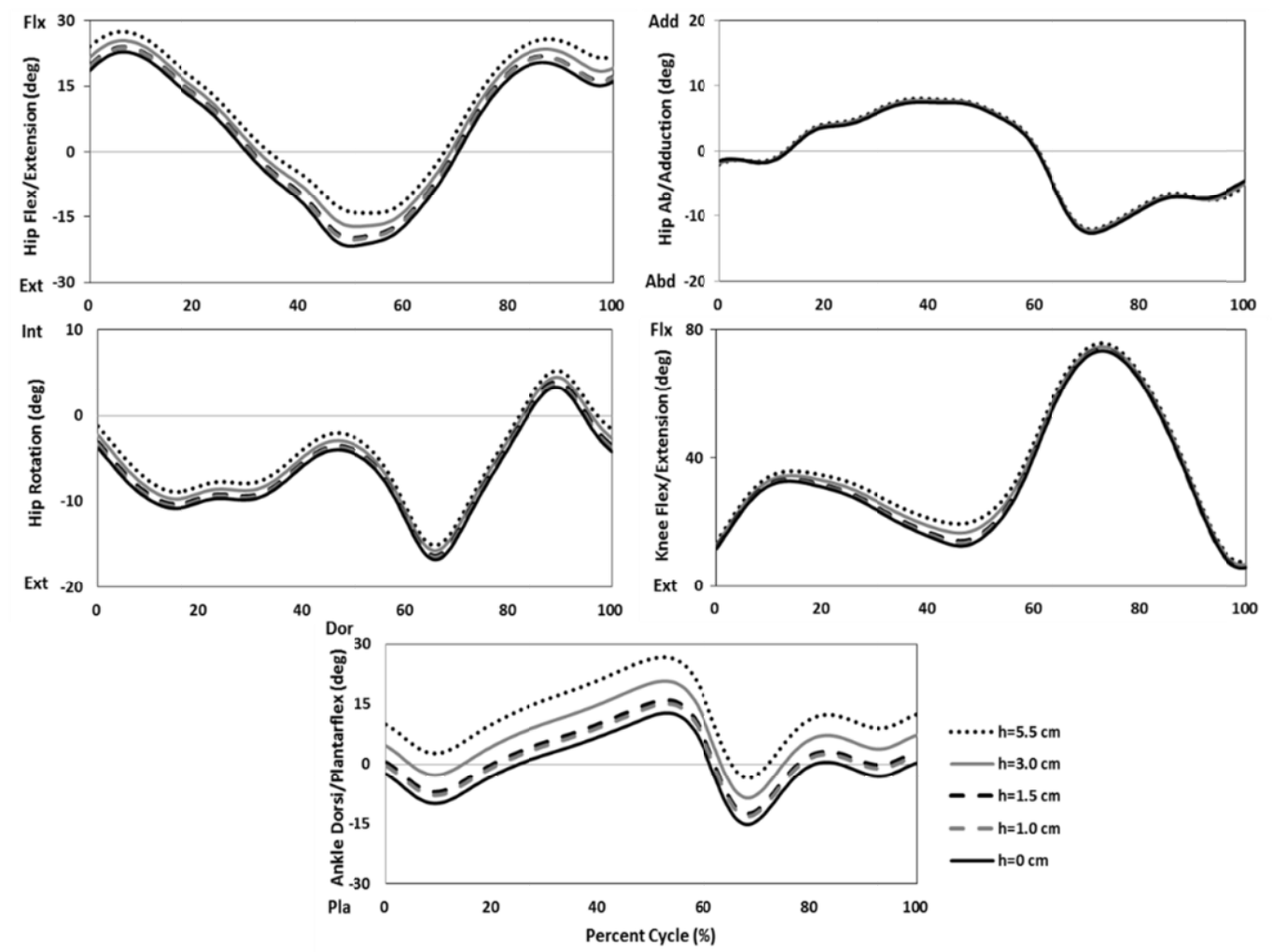

Fig. 2. A representative lower limb joint kinematics by toe marker placement error for one subject.

$$
N R M S E=\frac{R M S E}{x_{\max }-x_{\min }} \times 100 \%
$$

Where

$$
R M S E=\sqrt{\frac{\sum_{i=1}^{101}\left(x_{1, i}-x_{2, i}\right)^{2}}{101}}
$$

$\mathrm{x}_{\max }$ and $\mathrm{x}_{\min }$ represented the maximum and minimum values in the normalized 101 data point of joint kinematics or muscle forces. $\mathrm{x}_{1, \mathrm{i}}$ and $\mathrm{x}_{2, \mathrm{i}}$ represented the corresponding normalized data point for reference position and four different height of joint kinematics or muscle force.

\section{Results and discussion}

The corresponding joint kinematic curves were similar except for ankle dorsiflexion/plantarflexion, which was layered for all three subjects, a representative lower limb joint kinematics by toe marker placement error shown in Figure 2. The results of joint kinematics and muscle force differences when comparing toe marker reference position were presented in Table 1. We found that the ankle 
dorsiflexion/plantarflexion was significantly different when comparing $\mathrm{h}=5.5 \mathrm{~cm}$ (NRMSE $=42.9 \%$ ), $\mathrm{h}=3.0 \mathrm{~cm}(\mathrm{NRMSE}=25.1 \%)$ and $\mathrm{h}=1.5 \mathrm{~cm}(\mathrm{NRMSE}=10.5 \%)$ with $\mathrm{h}=0 \mathrm{~cm}$. The hip and knee joint kinematics were not significantly influenced by toe marker placement error except for hip flexion/extension when $\mathrm{h}=5.5 \mathrm{~cm}(\mathrm{NRMSE}=13.5 \%)$. Five of sixteen muscle forces were different when comparing $\mathrm{h}=5.5 \mathrm{~cm}$ with $\mathrm{h}=0 \mathrm{~cm}$. These muscles were iliacus (NRMSE=16.2\%), psoas $(\mathrm{NRMSE}=16.8 \%$ ), rectus femoris $(\mathrm{NRMSE}=12.7 \%)$, soleus (NRMSE=12.4\%), and tibialis posterior $(\mathrm{NRMSE}=17.8 \%$ ).

The results showed clearly that toe marker placement error affected hip, knee and ankle joint kinematics using OpenSim simulation. Although the hip and knee joint kinematics were mainly determined by the markers located on the thigh and shank, other markers and the weighting of markers still played a function in these joints since all joint kinematics were determined together and have interaction in OpenSim. Therefore, theoretically, toe marker placement error affected all the joint kinematics though the magnitudes varied significantly by joints. The residual variances for joint kinematics had an approximately linear relationship with toe marker placement error for lower limb joints, which was shown in Figure 3. The ankle joint kinematics were more sensitive to the toe marker placement error as expected since toe marker directly involved to determine the ankle joint motion in the sagittal plane. The residual variance was relatively larger for hip flexion/extension and rotation than hip abduction/adduction and knee flexion/extension. That may explain by the relative large motion range for hip joint motion in the sagittal and transverse planes than the frontal plane. But the phenomenon of small knee flexion/extension was still unclear.

Table 1

Joint kinematics and muscle force differences when comparing toe marker reference position

\begin{tabular}{|c|c|c|c|c|}
\hline joint kinematics and muscle force & $\begin{array}{l}\text { NRMSE } \\
\mathrm{h}=5.5 \mathrm{~cm}\end{array}$ & $\mathrm{~h}=3.0 \mathrm{~cm}$ & $\mathrm{~h}=1.5 \mathrm{~cm}$ & $\mathrm{~h}=1 \mathrm{~cm}$ \\
\hline hip flexion/extension & $13.5 \% *$ & $7.6 \%$ & $3.3 \%$ & $2.5 \%$ \\
\hline hip abduction/adduction & $2.5 \%$ & $1.3 \%$ & $0.5 \%$ & $0.6 \%$ \\
\hline hip rotation & $9.6 \%$ & $5.6 \%$ & $2.7 \%$ & $1.6 \%$ \\
\hline knee flexion/extension & $5.6 \%$ & $3.2 \%$ & $1.3 \%$ & $0.7 \%$ \\
\hline ankle dorsiflexion/plantarflexion & $42.9 \% *$ & $25.1 \% *$ & $10.5 \% *$ & $7.8 \%$ \\
\hline gluteus maximus1 & $5.3 \%$ & $3.1 \%$ & $1.9 \%$ & $1.5 \%$ \\
\hline gluteus maximus2 & $4.6 \%$ & $3.0 \%$ & $1.9 \%$ & $1.6 \%$ \\
\hline gluteus medius 1 & $3.9 \%$ & $2.4 \%$ & $1.0 \%$ & $0.8 \%$ \\
\hline gluteus medius 2 & $2.9 \%$ & $2.1 \%$ & $1.2 \%$ & $1.2 \%$ \\
\hline gluteus medius 3 & $6.2 \%$ & $4.0 \%$ & $2.2 \%$ & $2.2 \%$ \\
\hline iliacus & $16.2 \% *$ & $8.6 \%$ & $3.1 \%$ & $2.3 \%$ \\
\hline psoas & $16.8 \% *$ & $8.4 \%$ & $3.1 \%$ & $2.4 \%$ \\
\hline adductor magnus & $9.0 \%$ & $5.9 \%$ & $1.9 \%$ & $1.7 \%$ \\
\hline biceps femoris long head & $5.1 \%$ & $3.0 \%$ & $1.4 \%$ & $1.0 \%$ \\
\hline biceps femoris short head & $6.4 \%$ & $3.9 \%$ & $1.9 \%$ & $1.4 \%$ \\
\hline rectus femoris & $12.7 \% *$ & $6.4 \%$ & $2.6 \%$ & $2.4 \%$ \\
\hline vastus intermedius & $8.6 \%$ & $4.9 \%$ & $1.8 \%$ & $1.1 \%$ \\
\hline soleus & $12.4 \% *$ & $9.4 \%$ & $4.6 \%$ & $4.0 \%$ \\
\hline gastrocnemius & $7.9 \%$ & $5.1 \%$ & $2.3 \%$ & $2.6 \%$ \\
\hline tibialis anterior & $5.3 \%$ & $3.8 \%$ & $1.5 \%$ & $1.2 \%$ \\
\hline tibialis posterior & $17.8 \% *$ & $9.5 \%$ & $3.6 \%$ & $2.8 \%$ \\
\hline
\end{tabular}

Note: $*$ indicated result was significant at the NRMSE $>10 \%$ level 


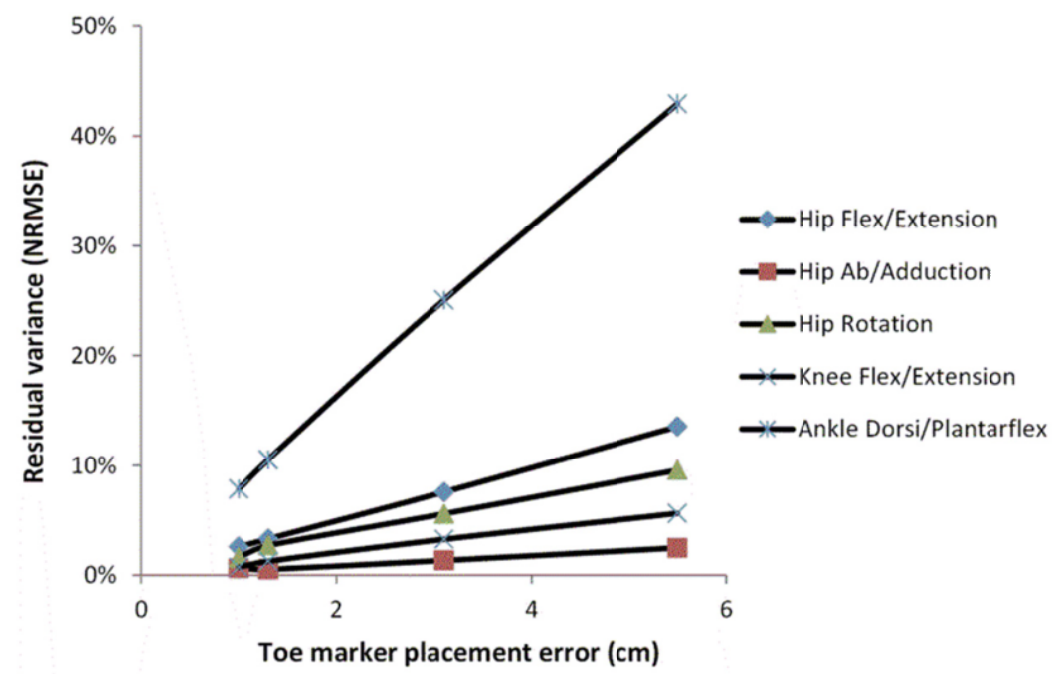

Fig. 3. The lower limb joint kinematics residual variance by toe marker placement error.

The muscle forces responded to the residual variance of joint kinematics to various degrees based on the muscle function for specific joint kinematics. The iliacus and psoas mainly responded to the change of hip rotation since they are the main functional muscles for hip external rotation. The significant force difference for rectus femoris balanced the additional knee joint torque generated by gastrocnemius, which crosses both knee and ankle joints. The force change of soleus and tibialis posterior responded the different ankle kinematics and prevent the model from falling.

Some limitations existed in the present study. First, the actual change of gait pattern due to the footwear was neglected. Previous research indicated that shoes restrict the natural motion of barefoot walking [14] and the texture of footwear influences ankle kinematics and muscle activity [15]. Second, three subjects were a small sample size, which potentially limited the generalizability of the results. Further study should increase the sample size to have more statistical power to testify the finding in the present study.

\section{Conclusions}

Skeleton model creation from marker-based motion tracking of anatomical landmarks has an important role in biomechanics and musculoskeletal simulation. This study demonstrates the importance of evaluating toe marker position error for the joint kinematics and predicted muscle forces. The presence of footwear or other occlusions that prevent accurate placement of reference and tracking markers should be considered when interpreting results derived from simulation software such as OpenSim. Further work should focus on generating more robust marker sets and modeling techniques that are less sensitive to these errors. 


\section{Acknowledgments}

This work was supported by University Science Research Project of Jiangsu Province (14KJB310022), Open Project of Jiangsu Province Keylab of Anesthesiology (KJS1401) and Scientific Research Foundation for Excellent Talents of Xuzhou Medical College (D2014018). The authors also would like to thank Dr. Kasey Greenland for his comments and useful discussions.

\section{References}

[1] L. Chiari, U.D. Croce, A. Leardini and A. Cappozzo, Human movement analysis using stereophotogrammetry: Part 2: Instrumental errors, Gait Posture 21 (2005), 197-211.

[2] A. Leardini, L. Chiari, U.D. Croce and A. Cappozzo, Human movement analysis using stereophotogrammetry: Part 3. Soft tissue artifact assessment and compensation, Gait Posture 21 (2005), 212-225.

[3] U. Della Croce, A. Leardini, L. Chiari and A. Cappozzo, Human movement analysis using stereophotogrammetry: Part 4: assessment of anatomical landmark misplacement and its effects on joint kinematics, Gait Posture 21 (2005), 226237.

[4] K.S. Al-Zahrani and M.O. Bakheit, A historical review of gait analysis, Neurosciences (Riyadh) 13 (2008), 105-108.

[5] G.E. Gorton III, D.A. Hebert and M.E. Gannotti, Assessment of the kinematic variability among 12 motion analysis laboratories, Gait Posture 29 (2009), 398-402.

[6] E. Szczerbik and M. Kalinowska, The influence of knee marker placement error on evaluation of gait kinematic parameters, Acta of Bioengineering \& Biomechanics 13 (2011), 43-46.

[7] P.D. O'Connor, M.E. Robinson, F.R. Shirley and M.M. Millan, The effect of marker placement deviations on spinal range of motion determined by video motion analysis, Physical Therapy 73 (1993), 478-483.

[8] L. France and C. Nester, Effect of errors in the identification of anatomical landmarks on the accuracy of Q angle values, Clinical Biomechanics (Bristol, Avon) 16 (2001), 710-713.

[9] G. Li, K. Kaufman, E. Chao and H. Rubash, Prediction of antagonistic muscle forces using inverse dynamic optimization during flexion/extension of the knee, Journal of Biomechanical Engineering 121 (1999), 316-322.

[10] B.J. Fregly, T.F. Besier, D.G. Lloyd, S.L. Delp, S.A. Banks, M.G. Pandy and D.D. D'Lima, Grand challenge competition to predict in vivo knee loads, Journal of Orthopaedic Research 30 (2012), 503-513.

[11] F.C. Anderson and M.G. Pandy, A dynamic optimization solution for vertical jumping in three dimensions, Computer Methods in Biomechanics and Biomedical Engineering 2 (1999), 201-231.

[12] D.G. Thelen and F.C. Anderson, Using computed muscle control to generate forward dynamic simulations of human walking from experimental data, Journal of Biomechanics 39 (2006), 1107-1115.

[13] G. Giakas and V. Baltzopoulos, Time and frequency domain analysis of ground reaction forces during walking: An investigation of variability and symmetry, Gait Posture 5 (1997), 189-197.

[14] C. Morio, M.J. Lake, N. Gueguen, G. Rao and L. Baly, The influence of footwear on foot motion during walking and running, Journal of Biomechanics 42 (2009), 2081-2088.

[15] M.A. Nurse, M. Hulliger, J.M. Wakeling, B.M. Nigg and D.J. Stefanyshyn, Changing the texture of footwear can alter gait patterns, Journal of Electromyography and Kinesiology 15 (2005), 496-506. 